\title{
Addition of a fixed combination of brinzolamide I\%/timolol $0.5 \%$ to prostaglandin monotherapy in patients with glaucoma or ocular hypertension
}

This article was published in the following Dove Press journal:

Clinical Ophthalmology

8 December 201 I

Number of times this article has been viewed

\author{
Katrin Lorenz' \\ Klaus Rosbach ${ }^{2}$ \\ Andreas Matt ${ }^{3}$ \\ Norbert Pfeiffer ${ }^{1}$ \\ 'University Medical Center, Johannes \\ Gutenberg-Universität Mainz, Mainz, \\ Germany; ${ }^{2}$ Private practice, Mainz, \\ Germany; ${ }^{3}$ Private practice, \\ Köln-Hohenhaus, Germany
}

Background: This study was conducted to evaluate the safety and efficacy of adding a fixed combination of brinzolamide $1 \% /$ timolol $0.5 \%$ to prostaglandin analog (PGA) monotherapy in patients with primary open-angle glaucoma, pigment dispersion glaucoma, or ocular hypertension who require additional intraocular pressure (IOP) reduction.

Methods: This was a prospective, multicenter $(n=5)$, open-label, single-arm, Phase IV clinical trial in which patients currently being treated with a PGA but requiring additional IOP reduction were administere brinzolamide $1 \%$ /timolol $0.5 \%$ twice daily as adjunctive therapy to their current PGA monotherapy regimen. The primary objective was to examine the IOP-lowering efficacy of brinzolamide-timolol when used as adjunctive therapy.

Results: Forty-seven patients enrolled in and completed the study. After 12 weeks of adjunctive brinzolamide-timolol therapy, the mean IOP of the total patient population decreased from $22.1 \mathrm{mmHg}$ at baseline to $16.7 \mathrm{mmHg}$. The mean IOP reduction of $5.4 \mathrm{mmHg}(24.4 \%)$ was both clinically and statistically significant $(P<0.001)$. This significant decrease in mean IOP at week 12 was maintained across all PGA groups $(P<0.05)$. No significant differences were observed in symptom frequency between baseline and week 12 for any of the six solicited symptoms. A total of 17 adverse events from six patients was reported, of which ten were drug-related. Most $(n=7)$ of the drug-related adverse events were mild or moderate in intensity. None of the adverse events required any treatment or resulted in treatment interruption or discontinuation. Of the 90 eligible eyes, $85.6 \%$ had a decrease in IOP of at least $3 \mathrm{mmHg}$ from baseline and $98 \%$ of patients had a decrease in IOP of $\geq 1 \mathrm{mmHg}$.

Conclusion: This study suggests that a fixed combination of brinzolamide $1 \% /$ timolol $0.5 \%$ can provide additional IOP reduction effectively and safely when used as adjunctive therapy for patients receiving insufficient IOP reduction from PGA monotherapy.

Keywords: adjunctive therapy, brinzolamide, carbonic anhydrase inhibitor, fixed combination, glaucoma, intraocular pressure

\section{Introduction}

Prostaglandin analogs (PGAs) are one of the most common first-line therapies used to reduce intraocular pressure (IOP). ${ }^{1}$ While PGAs can reduce IOP by approximately $30 \%(6-8 \mathrm{mmHg}),{ }^{2-4}$ patients will often require a further decrease to achieve an individually set target IOP. In such cases, a stepwise approach is typically used in which a single hypotensive agent, such as a beta-blocker, a carbonic anhydrase inhibitor, or an alpha-agonist, is added to therapy. ${ }^{1}$ Replacing the PGA with a fixed combination of a PGA + a beta-blocker has produced an additional $13.7 \%-22.6 \%$ decrease in IOP compared with PGA monotherapy alone. ${ }^{5,6}$ However, other fixed combinations have also been used as adjunctive therapy, effectively progressing a patient directly to a three-drug regimen.
Correspondence: Katrin Lorenz

University Medical Center, Johannes

Gutenberg-Universität Mainz,

Langenbeckstrasse I, 55I3I Mainz,

Germany

Tel +49 6I3II7 4069

Fax +49613117474069

Email katrin.lorenz@unimedizin-mainz.de 
Brinzolamide 1\%/timolol 0.5\% (Azarga ${ }^{\circledR}$, Alcon Pharma $\mathrm{GmbH}$, Puurs, Belgium) is a fixed-combination product that combines a carbonic anhydrase inhibitor with a beta-blocker. Brinzolamide-timolol was recently approved in the European Union for the treatment of primary open-angle glaucoma or ocular hypertension in patients for whom monotherapy provides insufficient IOP reduction. ${ }^{7}$ Brinzolamide-timolol has been shown to produce both statistically and clinically superior reductions in IOP from baseline $(8.0-8.7 \mathrm{mmHg})$ compared with either brinzolamide $1 \%$ alone $(5.1-5.6 \mathrm{mmHg})$ or timolol $0.5 \%$ alone $(5.7-6.9 \mathrm{mmHg}){ }^{8}$ It has also demonstrated noninferior IOP-lowering efficacy and less ocular discomfort when compared with a fixed combination of dorzolamide $2 \% /$ timolol $0.5 \%$ in patients with open-angle glaucoma or ocular hypertension. ${ }^{9,10}$ However, since clinical data on the use of brinzolamide-timolol as adjunctive therapy are lacking, a study was conducted to evaluate the safety and efficacy of adding brinzolamide-timolol to PGA monotherapy in patients with either primary open-angle glaucoma, pigment dispersion glaucoma, or ocular hypertension who require further IOP reduction.

\section{Methods}

This was a prospective, multicenter (five sites throughout Germany), open-label, single-arm Phase IV clinical trial in which patients currently being treated with a PGA but requiring additional reduction in IOP were administered brinzolamide $1 \% /$ timolol $0.5 \%$ twice daily in addition to their current PGA monotherapy regimen. The protocol was approved by the ethics committee of the Landesärztekammer Rheinland Pfalz in Mainz and the study was performed in compliance with the ethical principles of the Declaration of Helsinki and Good Clinical Practice. All participating patients provided their written informed consent.

\section{Patients}

Eligible patients were at least 21 years old with a diagnosis of primary open-angle glaucoma, pigment dispersion glaucoma, or ocular hypertension in at least one eye. If both eyes qualified for the study, both were treated and analyzed using an average eye analysis. Patients must have been treated with PGA monotherapy for at least 4 weeks prior to visit 1 (baseline visit), and IOP had to be at least $20 \mathrm{mmHg}$ in the qualifying eye(s) but no more than $35 \mathrm{mmHg}$ in both eyes while receiving treatment with PGA monotherapy. IOP in both eyes had to be considered safe by the investigator to assure clinical stability of vision and the optic nerve throughout the duration of the trial. Patients were required to have a best-corrected Snellen visual acuity of at least 20/200 in both eyes.

Patients were excluded if they met any of the following criteria: presence of any primary or secondary glaucoma not listed in the inclusion criteria; presence of extreme narrow angle with complete or partial closure in either eye, as measured by gonioscopy; any abnormality preventing reliable applanation tonometry in qualifying eye(s); presence of corneal dystrophies; any opacity or patient uncooperativeness that restricts adequate examination of the ocular fundus or anterior chamber of either eye; concurrent infectious/ noninfectious conjunctivitis, keratitis or uveitis in either eye; intraocular conventional surgery or laser surgery in qualifying eye(s) less than 3 months prior to visit 1; risk of visual field or visual acuity worsening as a consequence of trial participation in the investigator's opinion; progressive retinal or optic nerve disease from any cause apart from glaucoma; women of childbearing potential not using reliable means of birth control or who were pregnant or lactating; any clinically significant, serious, or severe medical or psychiatric condition; a condition, which, in the opinion of the investigator, would interfere with trial participation or would present a significant risk to the patient; participation in any other investigational study within 30 days prior to visit 1 ; use of systemic medications known to affect IOP (eg, oral beta-adrenergic blockers, alpha-agonists and alpha-blockers, angiotensinconverting enzyme inhibitors, and calcium channel blockers) without being changed for at least 7 days prior to visit 1 or an anticipated change in the dosage/regimen during the course of the study; use of systemic carbonic anhydrase inhibitors (eg, methazolamide, acetazolamide); current or anticipated use of systemic corticosteroids, by any route except inhaled, for longer than 2 weeks during the trial; severe allergic rhinitis; hypersensitivity to beta-blockers other than timolol; severely impaired renal function; hyperchloremic acidosis; bronchial asthma or a history of bronchial asthma, bronchial hyperreactivity, or severe chronic obstructive pulmonary disease that would preclude the safe administration of a topical beta-blocker; sinus bradycardia ( $<55 \mathrm{bpm})$, second-degree or third-degree atrioventricular block, sinoatrial block, overt cardiac failure, or cardiogenic shock that would preclude safe administration of a topical beta-blocker; history of myasthenia gravis, allergy to sulfur, ocular herpes simplex, allergy or sensitivity to any components of the preparations to be used in this trial deemed clinically significant in the opinion of the investigator; history of, or at risk for, uveitis or cystoid macular edema; or unwillingness to accept the risk of iris, skin, or eyelash changes associated with PGA therapy. 


\section{Study design}

At baseline (visit 1), patients underwent Goldmann applanation tonometry, best-corrected visual acuity testing (Snellen equivalent), and slit lamp biomicroscopy. For patients who were eligible for trial participation, both solicited and unsolicited adverse events were recorded and the solicited symptom survey was administered. The symptom survey queried patients about six common ocular symptoms, ie, light sensitivity, blurred or dim vision, stinging or burning, foreign body sensation, pain, and redness. Patients were instructed to administer brinzolamide-timolol twice daily ( 8 am and $8 \mathrm{pm}$ ) for 12 weeks in addition to their current PGA monotherapy, with the two medications taken at least 5 minutes apart. Week 4 (safety) and week 12 (efficacy) visits were scheduled within 1 hour of the baseline visit. At the week 4 and week 12 visits, Goldmann applanation tonometry, best-corrected visual acuity testing (Snellen equivalent), and slit lamp biomicroscopy were conducted, and both solicited and unsolicited adverse events were recorded. In addition, at the week 12 visit, the solicited symptom survey was administered, including additional questions about the duration of symptoms and tolerability of the study medication. Treatment success was assessed by the investigator at the week 12 visit.

\section{Statistical analysis}

The primary outcome measure was mean IOP change at week 12 for the total study population and was analyzed by a one-way analysis of variance test. The study provided a $90 \%$ power to detect a difference of $1.5 \mathrm{mmHg}$ between baseline IOP and week 12 IOP if at least 40 patients were analyzed. The secondary outcome measure was the mean IOP change at week 12 across patient PGA subgroups (latanoprost, travoprost, bimatoprost, and tafluprost), which was analyzed by a paired $t$-test within a one-way analysis of variance test. The study provided an $80 \%$ power to detect a difference of $2.0 \mathrm{mmHg}$ among subgroups that contain at least 17 patients. One-way analysis of variance tests were used to analyze visual acuity differences and solicited symptom survey differences. All data analyses were twosided and used the intent-to-treat population. If both eyes qualified for the study, both were treated and analyzed using an average eye analysis. If an ineligible eye was treated with study medication, that eye was included in the safety analysis but not included in the efficacy analysis. An $\alpha$-level of 0.05 was used to determine statistical significance. The data were analyzed by PRN Pharmaceutical Research Network, LLC (Dallas, TX).
Table I Patient demographics $(n=47)$

\begin{tabular}{ll}
\hline Demographics & $\mathbf{n}(\%)$ \\
\hline Age (years) & \\
$\leq 55$ & $8(17)$ \\
$56-65$ & $15(32)$ \\
$66-75$ & $16(34)$ \\
$\geq 76$ & $8(17)$ \\
Gender & \\
Male & $16(34)$ \\
Female & $31(66)$ \\
Race & \\
Caucasian & $47(100)$ \\
Qualified eye(s) & \\
OU & $43(91)$ \\
OD & $3(6)$ \\
OS & $1(2)$ \\
Diagnosis & \\
Primary open-angle glaucoma & $44(94)$ \\
Ocular hypertension & $3(6)$ \\
Prostaglandin analog therapy & \\
Latanoprost $0.005 \%$ & $20(43)$ \\
Travoprost $0.004 \%$ & $20(43)$ \\
Bimatoprost $0.03 \%$ & $4(9)$ \\
Tafluprost $0.0015 \%$ & $3(6)$ \\
\hline
\end{tabular}

\section{Results}

As shown in Table 1, 47 patients (90 eyes) were enrolled in the study. All participants were Caucasians. Mean age was 65.8 (range 31-92) years, with nearly all patients $(n=44)$ having a diagnosis of primary open-angle glaucoma and receiving bilateral study medication $(n=43)$. Most patients were taking either latanoprost $0.005 \%(\mathrm{n}=20)$ or travoprost $0.004 \%(\mathrm{n}=20)$ monotherapy at study enrollment. After 12 weeks of adjunctive brinzolamide-timolol therapy, the mean IOP of the total patient population decreased from

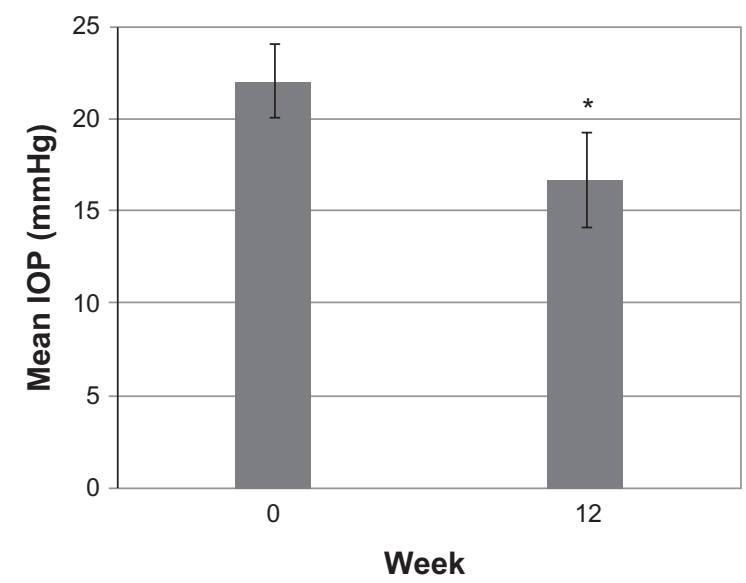

Figure I Mean intraocular pressure \pm standard deviation of total population across visits $(n=47)$.

Note: $* P<0.001$, week 12 vs week 0 (baseline). 


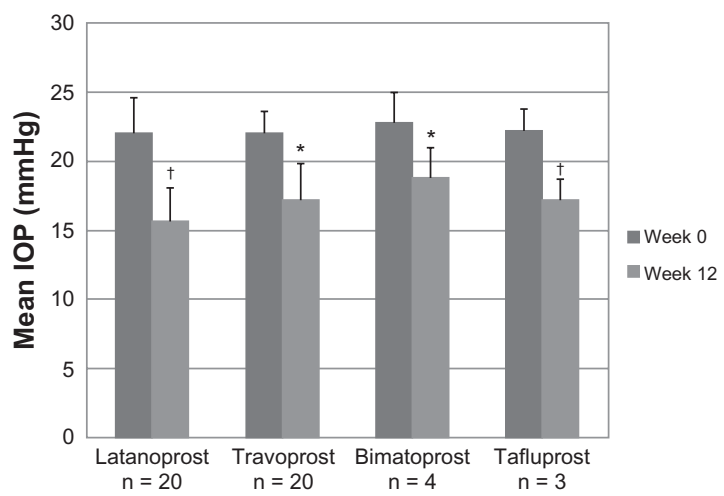

Figure 2 Mean intraocular pressure \pm standard deviation of prostaglandin analog therapy groups across visits $(n=47)$.

Notes: ${ }^{\dagger} P<0.05$, week 12 vs week 0 (baseline); $* P<0.00$ I, week 12 vs week 0 (baseline).

$22.1 \mathrm{mmHg}$ at baseline to $16.7 \mathrm{mmHg}$. This mean IOP reduction of $5.4 \mathrm{mmHg}(24.4 \%)$ was both clinically and statistically significant $(P<0.001$; Figure 1$)$. This decrease in mean IOP at week 12 was maintained across all PGA therapy groups $(P<0.05$; Figure 2$)$. Of the 90 eligible eyes, $85.6 \%$ had $\geq 3 \mathrm{mmHg}$ improvement in IOP from baseline. Ninety-eight percent of patients (46/47) had a $\geq 1 \mathrm{mmHg}$ reduction in IOP from baseline to week 12 and adequate tolerability.

No significant differences were observed in symptom frequency from baseline to week 12 for any of the six symptoms solicited (Figure 3). No significant differences were noted upon slit lamp biomicroscopy or visual acuity testing. A total of 17 adverse events from six patients was reported, of which 10 were drug-related (Table 2). One adverse event, gastroenteropathy, was classified as serious but was not considered to be related to the study drug. The other adverse events deemed not to be related to study drug were conjunctival hyperemia $(\mathrm{n}=2)$, and one each of beginning chalazion on upper lid, Helicobacter pylori infection, rash under the eye, and anxiety attack; all of these adverse events were mild or moderate in intensity. Most $(n=7)$ of the drug-related adverse events were mild or moderate in intensity; the severe events were one each of bitter taste, burning eyes, and blurred vision. None of the drug-related adverse events required any treatment, nor did any result in study drug interruption or discontinuation.

\section{Discussion}

In the current study, patients who were in need of additional ocular hypotensive therapy despite PGA monotherapy demonstrated a significant reduction in mean IOP with an adjunctive fixed combination of brinzolamide-timolol. This clinically and statistically significant IOP reduction was observed regardless of the type of concurrent PGA monotherapy. The fixed combination of brinzolamide-timolol alone was shown to produce an approximately $30 \%$ reduction in IOP (7-9 mmHg) in patients with untreated ocular hypertension or glaucoma. ${ }^{7}$ In this study, it produced a further mean reduction of $5.4 \mathrm{mmHg}(24.4 \%)$ when added to PGA monotherapy, demonstrating its usefulness as adjunctive hypotensive therapy. This activity is higher than that reported in previous studies of single-agent adjunctive therapy, in which mean IOP decreased by $5 \%-21 \%,{ }^{11,12}$ and is within the range of other adjunctive fixed combination therapies $(23.5 \%-29.3 \%){ }^{13}$

In general, the addition of two hypotensive agents as a fixed combination to PGA monotherapy was well tolerated in

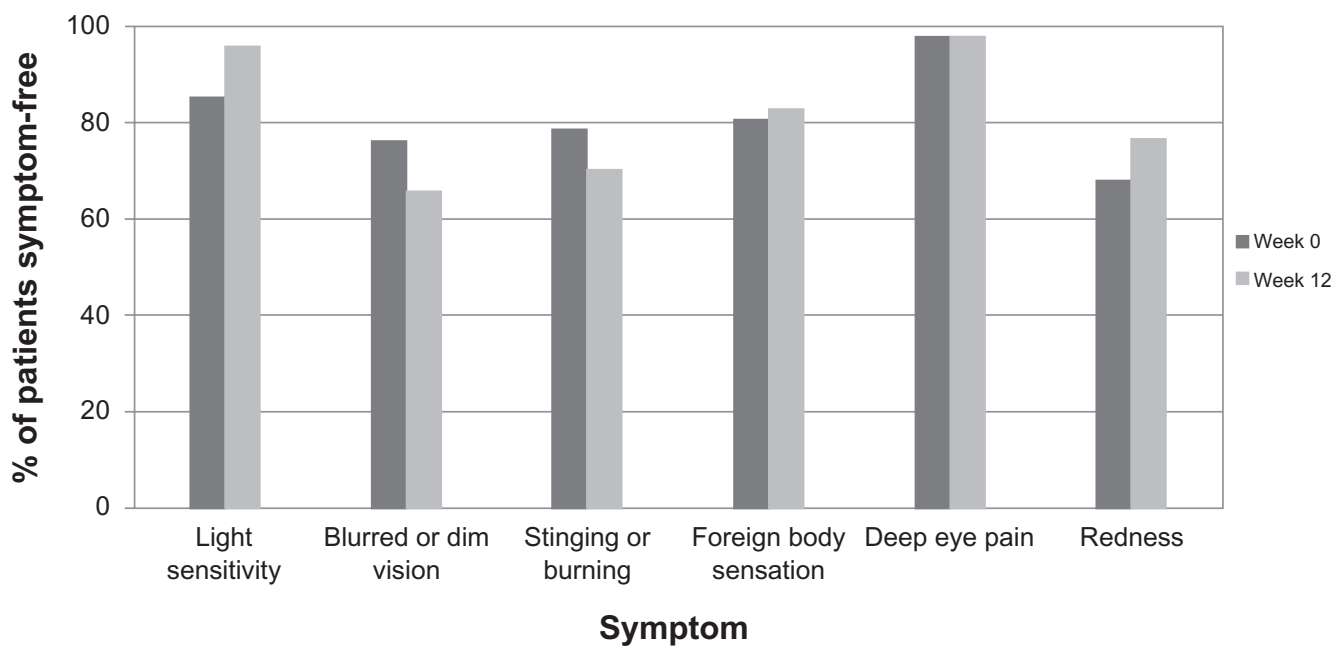

Figure 3 Symptom-free frequency at baseline and week $12(n=47)$. 
Table 2 Adverse events related to brinzolamide-timolol $(n=10)$

\section{Adverse event}

Bitter taste

Burning eye

Blurred vision

Dry eye

Glued eye

this study. No significant increase in symptom frequency was reported at week 12 with the symptom survey, which queried light sensitivity, burning/stinging, eye pain, redness, foreign body sensation, and blurred/dim vision. Drug-related adverse events were not anticipated, based on the well-known safety profiles of brinzolamide and timolol monotherapies. ${ }^{14,15}$ Furthermore, the incidence of these adverse events was similar to those reported previously with brinzolamidetimolol use. ${ }^{7}$ Adverse events were mostly mild or moderate. Although three of the 10 drug-related adverse events were severe, none resulted in study drug interruption, discontinuation, or required additional treatment. These symptom results and safety profile suggest that brinzolamide-timolol can be added to PGA monotherapy safely.

As with any clinical study, this trial did have some limitations which need to be kept in mind when interpreting its results. The design of this transition trial may have allowed for an imbalance in compliance with the two therapeutic regimens. Studies have demonstrated that drug compliance within clinical studies is frequently better than drug compliance in the "real world". ${ }^{16}$ Thus, the fact that the control medication (prior PGA monotherapy) was used outside the context of the clinical trial allows for the possibility that patients during the clinical study (when they used brinzolamide-timolol + PGA) may have exhibited increased compliance with that medication regimen than with their PGA monotherapy. An increase in compliance during the study could improve the efficacy of the adjunctive therapy. We used a rather modest definition of treatment success $(\geq 1 \mathrm{mmHg}$ reduction in IOP with adjunctive therapy). Although $98 \%$ of patients were classified as treatment successes, some of these patients may have achieved a decrease in IOP of only marginal clinical relevance. However, it is worth noting that more than $85 \%$ of the 90 treated eyes demonstrated at least a $3 \mathrm{mmHg}$ reduction in IOP with the addition of brinzolamide-timolol. Finally, with respect to the safety and tolerability of adjunctive brinzolamide-timolol, the short, 3-month duration of the study and the limited scope of the solicited symptom survey prevent conclusions as to its long-term efficacy and safety.
Nonetheless, this clinical study supports the hypothesis that brinzolamide $1 \%$ /timolol $0.5 \%$ is an effective adjunctive therapy when added to a prostaglandin analog in patients receiving insufficient IOP reduction from hypotensive monotherapy.

\section{Acknowledgments}

KL was supported in part by a grant from the "Clinical Trial Centers FKN 01KN1103, IZKS Mainz", the German Federal Ministry of Education and Research. Jennifer Klem provided medical writing support, which was funded by Alcon Laboratories Inc. Munir Marcel Keilani performed examinations and assisted with recruitment of patients.

\section{Disclosure}

NP has served on an advisory board for Alcon, and received an educational grant. The other investigators have no conflicts of interest to report in this work. This study was partially presented at the European Society of Ophthalmology/ American Academy of Ophthalmology in Geneva 2011 and at the World Glaucoma Congress in Paris 2011.

\section{References}

1. European Glaucoma Society. Terminology and Guidelines for Glaucoma. 3rd ed. Savona, Italy: Dogma; 2008.

2. Travatan [package insert]. Fort Worth, TX: Alcon Laboratories Inc; 2004.

3. Xalatan [package insert]. New York, NY: Pfizer Inc; 2009.

4. Lumigan [package insert]. Irvine, CA: Allergan Inc; 2010.

5. Centofanti M, Oddone F, Vetrugno M, et al. Efficacy of the fixed combinations of bimatoprost or latanoprost plus timolol in patients uncontrolled with prostaglandin monotherapy: a multicenter, randomized, investigator-masked, clinical study. Eur J Ophthalmol. 2009;19: 66-71.

6. Feuerhake C, Buchholz P, Kimmich F. Efficacy, tolerability and safety of the fixed combination of bimatoprost $0.03 \%$ and timolol $0.5 \%$ in a broad patient population: multicenter, open-label observational study. Curr Med Res Opin. 2009;25:1037-1043.

7. Azarga. Summary of product characteristics. Chicago, IL: Alcon Pharma; 2008.

8. Kaback M, Scoper SV, Arzeno G, et al. Intraocular pressure-lowering efficacy of brinzolamide $1 \% /$ timolol $0.5 \%$ fixed combination compared with brinzolamide $1 \%$ and timolol $0.5 \%$. Ophthalmology. 2008;115: $1728-1734$.

9. Manni G, Denis P, Chew P, et al. The safety and efficacy of brinzolamide $1 \% /$ timolol $0.5 \%$ fixed combination versus dorzolamide $2 \% /$ timolol $0.5 \%$ in patients with open-angle glaucoma or ocular hypertension. J Glaucoma. 2009;18:293-300.

10. Vold SD, Evans RM, Stewart RH, Walters T, Mallick S. A one-week comfort study of BID-dosed brinzolamide $1 \% /$ timolol $0.5 \%$ ophthalmic suspension fixed combination compared to BID-dosed dorzolamide $2 \% /$ timolol $0.5 \%$ ophthalmic solution in patients with open-angle glaucoma or ocular hypertension. J Ocul Pharmacol Ther. 2008;24: 601-605.

11. Bournias TE, Lai J. Brimonidine tartrate $0.15 \%$, dorzolamide hydrochloride $2 \%$, and brinzolamide $1 \%$ compared as adjunctive therapy to prostaglandin analogs. Ophthalmology. 2009;116:1719-1724. 
12. Cheng JW, Li Y, Wei RL. Systematic review of intraocular pressurelowering effects of adjunctive medications added to latanoprost. Ophthalmic Res. 2009;42:99-105.

13. Nixon DR, Yan DB, Chartrand JP, Piemontesi RL, Simonyi S, Hollander DA. Three-month, randomized, parallel-group comparison of brimonidine-timolol versus dorzolamide-timolol fixed-combination therapy. Curr Med Res Opin. 2009;25:1645-1653.
14. Azopt [package insert]. Fort Worth, TX: Alcon Laboratories Inc; 2008.

15. Timolol maleate ophthalmic solution USP [package insert]. Fort Worth, TX: Alcon Laboratories, Inc; 2004.

16. Andrade S. Compliance in the real world. Value Health. 1998;1: 171-173.

\section{Publish your work in this journal}

Clinical Ophthalmology is an international, peer-reviewed journal covering all subspecialties within ophthalmology. Key topics include: Optometry; Visual science; Pharmacology and drug therapy in eye diseases; Basic Sciences; Primary and Secondary eye care; Patient Safety and Quality of Care Improvements. This journal is indexed on
PubMed Central and CAS, and is the official journal of The Society of Clinical Ophthalmology (SCO). The manuscript management system is completely online and includes a very quick and fair peer-review system, which is all easy to use. Visit http://www.dovepress.com/ testimonials.php to read real quotes from published authors. 